\title{
Properties of the singing comet waves in the 67P/Churyumov-Gerasimenko plasma environment as observed by the Rosetta mission
}

\author{
H. Breuillard ${ }^{1,2}$, P. Henri ${ }^{1}$, L. Bucciantini ${ }^{1}$, M. Volwerk ${ }^{3}$, T. Karlsson ${ }^{4}$, A. Eriksson ${ }^{5}$, F. Johansson ${ }^{5}$, \\ E. Odelstad ${ }^{5}$, I. Richter ${ }^{6}$, C. Goetz ${ }^{6}$, X. Vallières ${ }^{1}$, and R. Hajra ${ }^{1,7}$ \\ ${ }^{1}$ Laboratoire de Physique et Chimie de l'Environnement et de l'Espace (LPC2E), UMR7328 CNRS/Université d'Orléans/CNES, \\ Orléans, France \\ e-mail: hugo.breuillard@cnrs-orleans.fr \\ 2 Laboratoire de Physique des Plasmas, UMR7648 CNRS/Ecole Polytechnique/Sorbonne University/University of Paris-Sud, \\ Paris, France \\ ${ }^{3}$ Space Research Institute, Austrian Academy of Sciences, Graz, Austria \\ ${ }^{4}$ KTH Royal Institute of Technology, Stockholm, Sweden \\ ${ }^{5}$ Swedish Institute of Space Physics, Uppsala, Sweden \\ ${ }^{6}$ Technical University of Braunschweig, Braunschweig, Germany \\ ${ }^{7}$ National Atmospheric Research Laboratory, Tirupati, India
}

Received 14 December 2018 / Accepted 29 May 2019

\begin{abstract}
Using in situ measurements from different instruments on board the Rosetta spacecraft, we investigate the properties of the newly discovered low-frequency oscillations, known as singing comet waves, that sometimes dominate the close plasma environment of comet 67P/Churyumov-Gerasimenko. These waves are thought to be generated by a modified ion-Weibel instability that grows due to a beam of water ions created by water molecules that outgass from the comet. We take advantage of a cometary outburst event that occurred on 2016 February 19 to probe this generation mechanism. We analyze the 3D magnetic field waveforms to infer the properties of the magnetic oscillations of the cometary ion waves. They are observed in the typical frequency range $(\sim 50 \mathrm{mHz})$ before the cometary outburst, but at $\sim 20 \mathrm{mHz}$ during the outburst. They are also observed to be elliptically right-hand polarized and to propagate rather closely $\left(\sim 0-50^{\circ}\right)$ to the background magnetic field. We also construct a density dataset with a high enough time resolution that allows us to study the plasma contribution to the ion cometary waves. The correlation between plasma and magnetic field variations associated with the waves indicates that they are mostly in phase before and during the outburst, which means that they are compressional waves. We therefore show that the measurements from multiple instruments are consistent with the modified ion-Weibel instability as the source of the singing comet wave activity. We also argue that the observed frequency of the singing comet waves could be a way to indirectly probe the strength of neutral plasma coupling in the 67P environment.
\end{abstract}

Key words. comets: general - comets: individual: 67P/Churyumov-Gerasimenko - plasmas - waves - methods: observational methods: data analysis

\section{Introduction}

For about two years, the groundbreaking ESA/Rosetta mission (Glassmeier et al. 2007a; Taylor et al. 2017) escorted comet 67P/Churyumov-Gerasimenko (hereafter 67P), while previous cometary missions were limited to flybys. For the first time, this enabled in situ measurements of the evolution of the ionized environment of a comet and its interaction with the incoming solar wind (Taylor et al. 2017). The plasma environment of 67P was probed by the Rosetta Plasma Consortium (hereafter RPC), which was a set of five instruments designed to monitor the plasma electrons, ions, and the electromagnetic field surrounding the comet (Carr et al. 2007). In particular, the magnetic field was observed by the Magnetometer (RPC-MAG, see Glassmeier et al. 2007b), and the plasma density was sometimes derived from two instruments: the Mutual Impedance Probe (RPC-MIP, see Trotignon et al. 2007) and the Langmuir probes (RPC-LAP, see Eriksson et al. 2007).
One of the most striking features discovered around 67P by Rosetta is a new type of low-frequency waves in a cometary environment (Richter et al. 2015), also referred to as "singing comet waves". They are quasi-coherent, large-amplitude $(\delta B / B=1)$, compressional magnetic field oscillations at $\sim 40 \mathrm{mHz}$ that dominate the close plasma environment of the cometary nucleus at large enough heliocentric distances ( $>2 \mathrm{AU}$ ) (see also, Richter et al. 2016). Richter et al. (2015) first suggested that these waves could arise from a cross-field current instability associated with currents carried by newborn cometary ions. Meier et al. (2016) then analytically discussed the modified ion-Weibel instability (Chang et al. 1990) as a possible source mechanism. Considering a cold homogeneous plasma composed of magnetized solar wind protons and electrons, they showed that a beam of unmagnetized water ions generates a cometary current. This configuration then drives a modified ion-Weibel instability that predominantly grows perpendicular to the current (Chang et al. 1990; Meier et al. 2016). The instability is expected at a frequency of about 
$f \sim 40 \mathrm{mHz}$ for the new water-ion mode in a cometary rest frame in compliance with the Rosetta observations from Richter et al. (2015). This result was later confirmed by Heinisch et al. (2017) using data from the lander Philae during touch-down on the surface on 2014 November 12. The Philae lander magnetometer ROMAP and the orbiter magnetometer RPC-MAG were both operating simultaneously at a roughly constant distance of $18.5 \mathrm{~km}$, which allowed for in situ magnetic two-point observations. The cometary ion mode was observed in the frequency range from 5 to $50 \mathrm{mHz}$ with an average frequency of about $8 \mathrm{mHz}$ at that time. It was also observed to propagate predominantly from the nucleus toward the Sun with a mean phase velocity of $\sim 5.3 \mathrm{~km} \mathrm{~s}^{-1}$ and a wavelength of $\sim 660 \mathrm{~km}$, in agreement with a modified ion-Weibel instability as a source (see also Glassmeier 2017).

In the frame of the modified ion-Weibel theory, Meier et al. (2016) found a maximum wave length of $\sim 980 \mathrm{~km}$ and phase velocities of about $v_{\mathrm{ph}} \sim 40 \mathrm{~km} \mathrm{~s}^{-1}$ perpendicular to the ambient magnetic field and the cometary current. They also showed that the frequencies of the water-ion mode only coincided with the proton gyro-frequency for small ambient magnetic fields $B_{0} \sim 1 \mathrm{nT}$, but were clearly distinguished for stronger fields. Finally, they demonstrated that the superposition of the strongest growing waves resulted in a fan-like phase structure close to the comet that yielded a wave length $<200 \mathrm{~km}$ with an apparent phase motion from the comet to the Sun, in agreement with two-point observations (Richter et al. 2016; Heinisch et al. 2017). These fan-like structures were also observed in the $+\mathrm{E}$ hemisphere of the environment of comet 67P in 3D hybrid simulations (Koenders et al. 2016). They were also attributed to a complex interference pattern triggered by a low-frequency wave activity at $\sim 95 \mathrm{mHz}$, in agreement with the frequency range found in Richter et al. (2015). Analyzing simulated fluctuations, the authors found that their magnetic polarization is predominantly right-handed, but neither purely transverse nor purely compressional.

Similar electromagnetic comet wave activity has also been observed during a cometary brightness outburst event, characterized by an increase in the coma surface brightness of two orders of magnitude, which occurred on 2016 February 19 at 10:00 UT (Grün et al. 2016). The onset of the outburst was preceded by pre-outburst decreases, observed in situ at Rosetta, in neutral gas density, in local plasma density, and in negative spacecraft potential at 09:50 UT. In response to the outburst, the neutral density increased by a factor of $\sim 2$ and the local plasma density increased by a factor of $\sim 3$. The local magnetic field exhibited a slight increase in amplitude ( $\sim 5 \mathrm{nT})$ and an abrupt rotation $\left(36.4^{\circ}\right)$ in response to the outburst (see Hajra et al. 2017). A weakening of $\sim 10-100 \mathrm{mHz}$ magnetic field fluctuations was also noted during the outburst, suggesting an alteration of the origin of the wave activity.

In this study, we make use of measurements from different RPC instruments to study the properties of the cometary ion mode observed before and during this cometary outburst, in order to probe their source mechanism. First, we perform a cross-calibration of RPC-MIP plasma density and RPC-LAP spacecraft potential variations using a simple spacecraft charging model to obtain plasma density measurements with a high enough time resolution to study the plasma contribution to the cometary waves (Sect. 2). Then, we analyze the 3D magnetic field waveform data from the RPC-MAG instrument to infer the properties of the magnetic oscillations (Sect. 3). Finally, we make use of these two datasets to investigate the correlations between plasma and magnetic field variations associated with the cometary ion mode (Sect. 4) before we conclude (Sect. 5).

\section{High time-resolution density measurements from RPC-MIP and RPC-LAP cross-calibration}

On the one hand, plasma densities estimated from the I-V curve resulting from sweeps of the Langmuir probe RPC-LAP were obtained about every $64 \mathrm{~s}$. On the other hand, electron densities estimated from the identification of the plasma frequency in the electric spectra of the Mutual Impedance Probe RPC-MIP were obtained every few seconds (2.5-32 s depending on operational modes). The time resolution of these two density datasets is hardly enough to resolve the characteristic timescales of the plasma oscillations associated with the electromagnetic waves generated at comet $67 \mathrm{P}$.

To overcome the low time resolution, densities can also be derived from RPC-LAP fixed-bias voltage or current, with a much higher time resolution (see Odelstad et al. 2018). However, these estimates suffer from the imposition of several physical assumptions, while densities derived from RPC-MIP measurements are believe to give a more reliable value of the absolute plasma density. Therefore, to benefit from the high time resolution of the density time series obtained from RPC-LAP voltage or current and the trustworthiness of RPC-MIP densities, we cross-calibrated the $17 \mathrm{~ms}$ (i.e., $60 \mathrm{~Hz}$ ) time-resolution RPCLAP floating potential measurements with RPC-MIP absolute density estimates and obtained a plasma density time series (hereafter called RPC-MIP/LAP density) with a time resolution sufficient for the analysis of plasma oscillations conducted in this paper.

For this purpose, we considered a simple charging model of the Rosetta orbiter where the incoming cometary electron current collected by the spacecraft body is balanced by the outgoing photoelectron current associated to the UV ionizing flux on the spacecraft, while the ion and secondary current contribution is considered negligible (Odelstad et al. 2015). This enabled us to express the spacecraft potential variations in terms of electron density variations:

$\ln \left(n_{\mathrm{e}}\right)=\ln \left(\frac{I_{\mathrm{ph}}}{e A_{\mathrm{S} / \mathrm{C}} \sqrt{\frac{k_{\mathrm{B}} T_{\mathrm{e}}}{2 \pi m_{\mathrm{e}}}}}\right)-\frac{e V_{\mathrm{S} / \mathrm{C}}}{\alpha k_{\mathrm{B}} T_{\mathrm{e}}}$,

where $n_{\mathrm{e}}$ is the electron plasma density, $I_{\mathrm{ph}}$ is the outgoing photoelectron current from the Rosetta spacecraft, $T_{\mathrm{e}}$ is the electron temperature, $A_{\mathrm{S} / \mathrm{C}}$ the spacecraft electron collection surface, $m_{\mathrm{e}}$ is the electron mass, $e$ is the electron charge, $k_{\mathrm{B}}$ is the Boltzmann constant, $V_{\mathrm{S} / \mathrm{C}}$ is the spacecraft potential with respect to the surrounding plasma, and $\alpha$ is a parameter that takes into account the position of the RPC-LAP Langmuir probes within the plasma sheath surrounding the Rosetta orbiter (Odelstad et al. 2017). To ensure that the spacecraft potential variations are associated with the variation in cometary plasma conditions without being contaminated by any other phenomenon, we have checked for the absence of spacecraft manoeuvres, such as thruster firings, wheels off-loading, or spacecraft attitude changes that could cause a variation in illumination conditions on the RPC-LAP Langmuir probes or on the Rosetta orbiter.

The RPC-MIP/RPC-LAP cross-calibration procedure assumes that the electron temperature variations occur on timescales much longer than for electron density variations. This is a reasonable assumption for phenomena on ion timescales, as the 


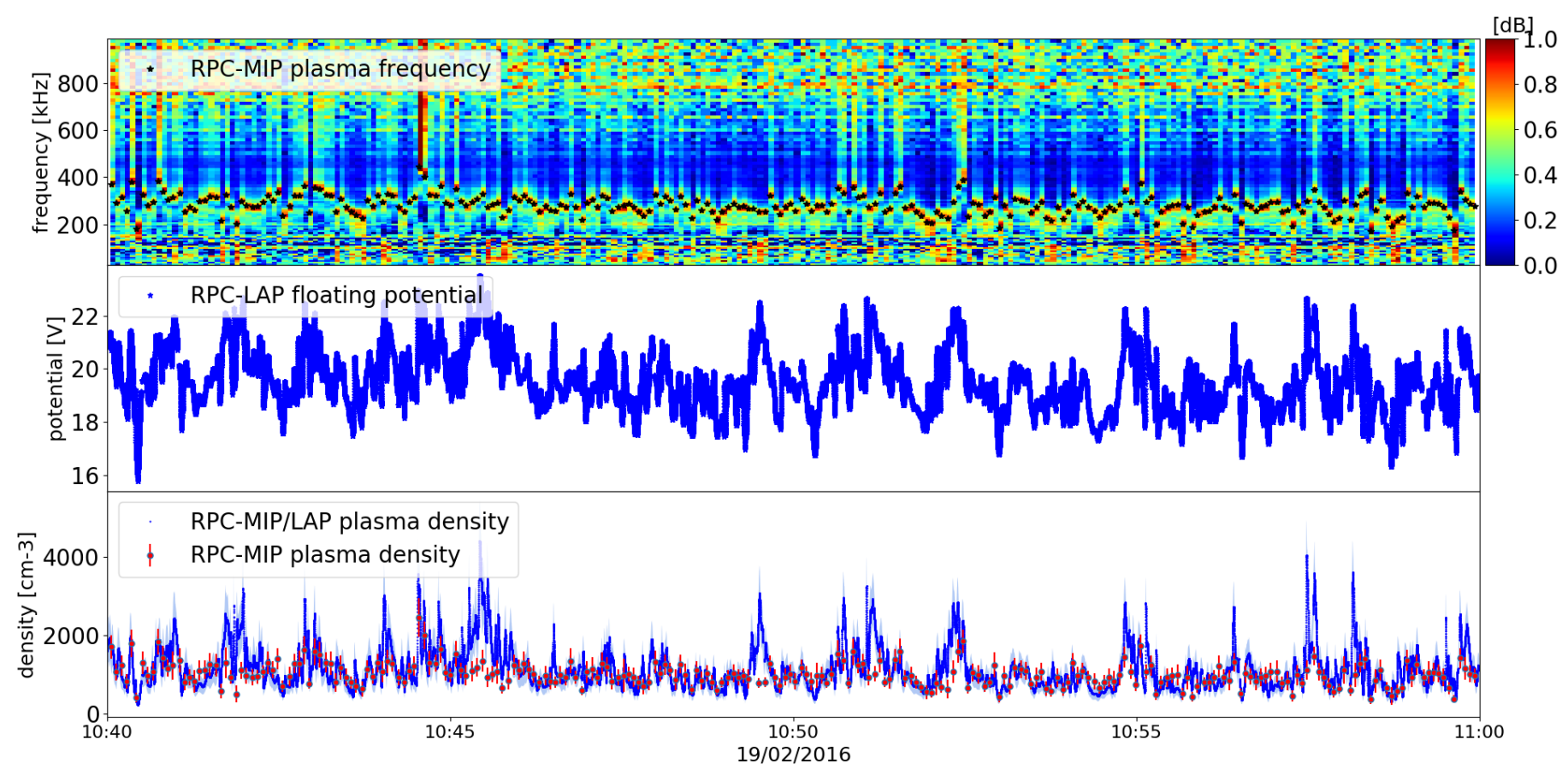

Fig. 1. Top panel: power amplitude of the mutual impedance spectrogram, normalized to that obtained in vacuum, expressed in $\mathrm{dB}$ (right color bar) in the [0-600] kHz frequency range. The black dots indicate the identified plasma frequency for each spectrum. Middle panel: electric potential measured by the Langmuir probe RPC-LAP1, operated in floating mode, with respect to spacecraft potential. Bottom panel: plasma densities extracted from the RPC-MIP mutual impedance spectra (black dots) together with the estimated maximum measurement errors (shaded gray region) and RPC-MIP/LAP plasma densities obtained from the cross-calibration of the RPC-LAP floating potential measurement with the RPC-MIP absolute density measurements (blue line).

rapidly moving electrons are able to reach a quasi-equilibrium at every time. It is also consistent with RPC-LAP electron temperature estimates, extracted from Langmuir sweeps analysis, which show much smaller relative temperature variations than the relative density variations observed by RPC-MIP or RPC-LAP during the Rosetta mission, at least during times when no strong electron cooling was observed, such as during the event studied in this work. At the same time, the photoelectron current from the spacecraft, dependent on the illuminated surface of the Rosetta orbiter, on the surface material properties, and on the heliocentric distance, is considered constant on timescales of about the density fluctuations. Practically, in this study we considered 20-min-long overlapping sliding windows, during which all parameters other than plasma density and spacecraft potential were assumed to remain constant. Inside each sliding window we applied a weighted orthogonal distance regression between the density time series retrieved from RPCMIP and the density time series derived from the RPC-LAP spacecraft potential time series using Eq. (1).

Uncertainties on the input dataset, namely (i) the RPCMIP plasma density and (ii) the RPC-LAP potential, are taken into account through the weight definition in the regression process. In the regression process, we used a subset of the two datasets: (i) RPC-MIP densities extracted from mutual impedance spectra characterized by a low signal-to-noise ratio (S/N) were discarded, and (ii) RPC-LAP potential measurements made during times that did not coincide with RPC-MIP density measurements (i.e., that did not fall in the RPC-MIP acquisition periods) were discarded. For each time window, best-fit parameters were then applied to the corresponding spacecraft potential time series. An average was then computed to merge the densities obtained in different overlapping time windows, and a cross-calibrated RPC-MIP/LAP plasma density time series was finally obtained, with $17 \mathrm{~ms}$ time resolution. The consistency between RPC-MIP plasma density measurements and RPCMIP/LAP cross-calibrated density estimates gives an a posteriori confirmation of the linearity between $\ln n_{\mathrm{e}}$ and $V_{\mathrm{S} / \mathrm{C}}$ (Eq. (1)) and that the isothermal electron evolution is a reasonable hypothesis. We also note that an isothermal evolution of the electrons at the frequency of the considered cometary waves was expected because the phase velocity of these cometary waves is much lower than the electron thermal velocity.

In the scope of this study, the cross-calibration procedure was performed for 2016 February 19. We note, however, that it was also applied to the whole cometary phase of the Rosetta mission and that the resulting cross-calibrated RPC-MIP/LAP densities are being archived in the publicly accessible ESA Planetary Science Archive (PSA).

The cross-calibration process is illustrated in Fig. 1 for 2016 February 19, where the input RPC-MIP and RPC-LAP time series (top two panels) as well as the output RPC-MIP/LAP cross-calibrated density (bottom panel) are shown. In particular, the top panel shows the values for the electron plasma frequencies (black dots), from which the RPC-MIP electron density was retrieved, on the RPC-MIP mutual impedance power spectra. The middle panel shows the input RPC-LAP floating potential measurements, from which the RPC-LAP spacecraft potential was derived. The bottom panel shows both the RPC-MIP/LAP cross-calibrated plasma densities (blue line) and the input RPC-MIP plasma densities (black dots) together with the associated uncertainties (gray shade).

Although most of the cross-calibrated data points in Fig. 1 fall into the uncertainties of MIP measurements, a few disagreements between the two datasets are sometimes observed 

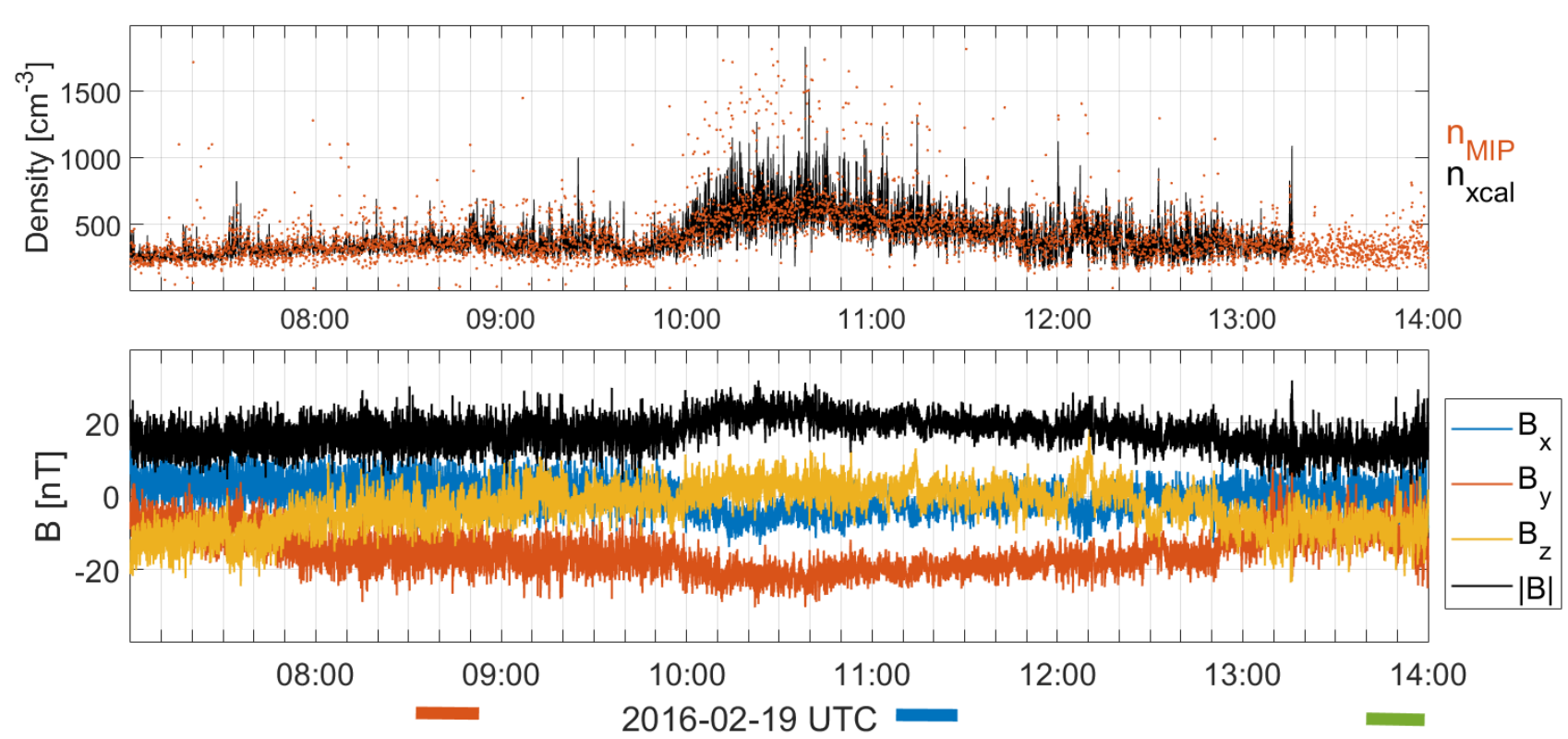

Fig. 2. Top panel: plasma electron density at the Rosetta orbiter in the time interval 07:00-14:00 of 2016 February 19. The black line indicates the RPC-MIP/LAP cross-calibrated plasma electron density while the orange dots represent the RPC-MIP plasma electron density. Bottom panel: RPC-MAG magnetic field components measured at the Rosetta orbiter in the time interval 07:00-14:00 of 2016 February 19. In particular, the $\mathrm{Bx}$ (blue line), By (orange line) and Bz (yellow line) components are shown together with the total magnetic field (black line). The 20-min time intervals used to compute Fig. 3 are shown as horizontal solid lines below the time tags, using the same color code.

(e.g., around 10:45). This reflects the fact that the crosscalibration model assumes a constant electron temperature over 20-min time windows. However, these discrepancies are observed to be on timescales (a few seconds to a few tens of seconds) much shorter than the timescales of interest in this study (singing comet waves are observed to have an average frequency of $50 \mathrm{mHz}$ ), and thus are not expected to affect our results.

We note that a similar but much simplified approach was used in the analysis of the final descent of Rosetta orbiter to comet 67P at the end of the mission operations on 2016 September 30 Heritier et al. (2017). However, a much lower final time resolution (from about $70 \mathrm{~ms}$ to $4 \mathrm{~s}$, varying in time) was used, assuming both isothermal electrons and constant photoelectron current collection at the spacecraft over the full-day descent instead.

Thanks to this high time-resolution of the RPC-MIP/LAP plasma densities, an investigation of the plasma oscillations associated with electromagnetic waves can be performed. In the following sections, after the analysis of magnetic field oscillations during the outburst event of 2016 February 19 at comet $67 \mathrm{P}$, the plasma pressure variations are investigated using these cross-calibrated densities.

\section{Magnetic-field oscillation properties from RPC-MAG measurements}

In this section, we study the cometary ion wave magnetic activity as measured by RPC-MAG on 2016 February 19 from 07:00 to 14:00 UT.

The raw 3D magnetic field waveform is shown in Fig. 2 in the comet-centered solar equatorial (CSEQ; see Acton 1996) coordinate system for the whole selected day. A sudden increase in the total B-field $\left(B_{\text {tot }}\right)$ at about 10:00 UTC can be distinguished, accompanied by a smoother decrease up to 14:00 UTC (see Grün et al. 2016) coincident with a cometary brightness outburst, whose impact on the cometary plasma is detailed in Hajra et al.

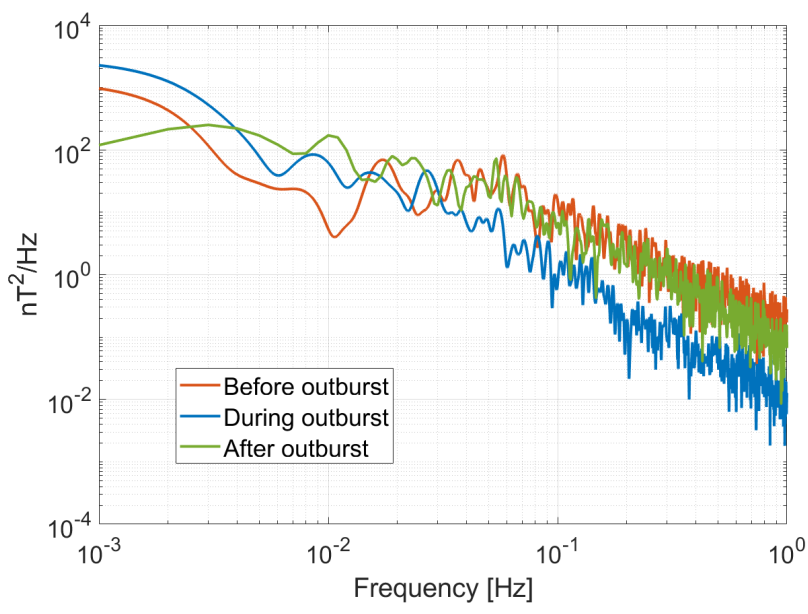

Fig. 3. Power spectral densities of the $B_{x}$ magnetic field component, estimated using Welch's method, for time windows of $20 \mathrm{~min}$ before (in orange), during (in blue), and after (in green) the outburst event observed at comet 67P at 10:00 UT on 2016 February 19.

(2017). In this paper, a significant weakening of the $10-100 \mathrm{mHz}$ magnetic wave activity in response to the outburst was reported.

We therefore used the magnetic field waveforms described above to study the power of the magnetic oscillations before and during the outburst that was observed at 10:00 UT. Figure 3 shows the power spectral density (PSD), estimated using Welch's method (Welch 1967). The 20-min intervals and the $B_{x}$ component were selected so that the PSDs can be compared to the results from Richter et al. (2015). In this figure, the magnetic power spectrum before and after the outburst clearly peaks at frequencies of 40-60 mHz, in good agreement with Richter et al. (2015), but during the outburst, it peaks at frequencies of $10-30 \mathrm{mHz}$. Although the magnetic PSD during the outburst is observed to be relatively higher than before the outburst in the 


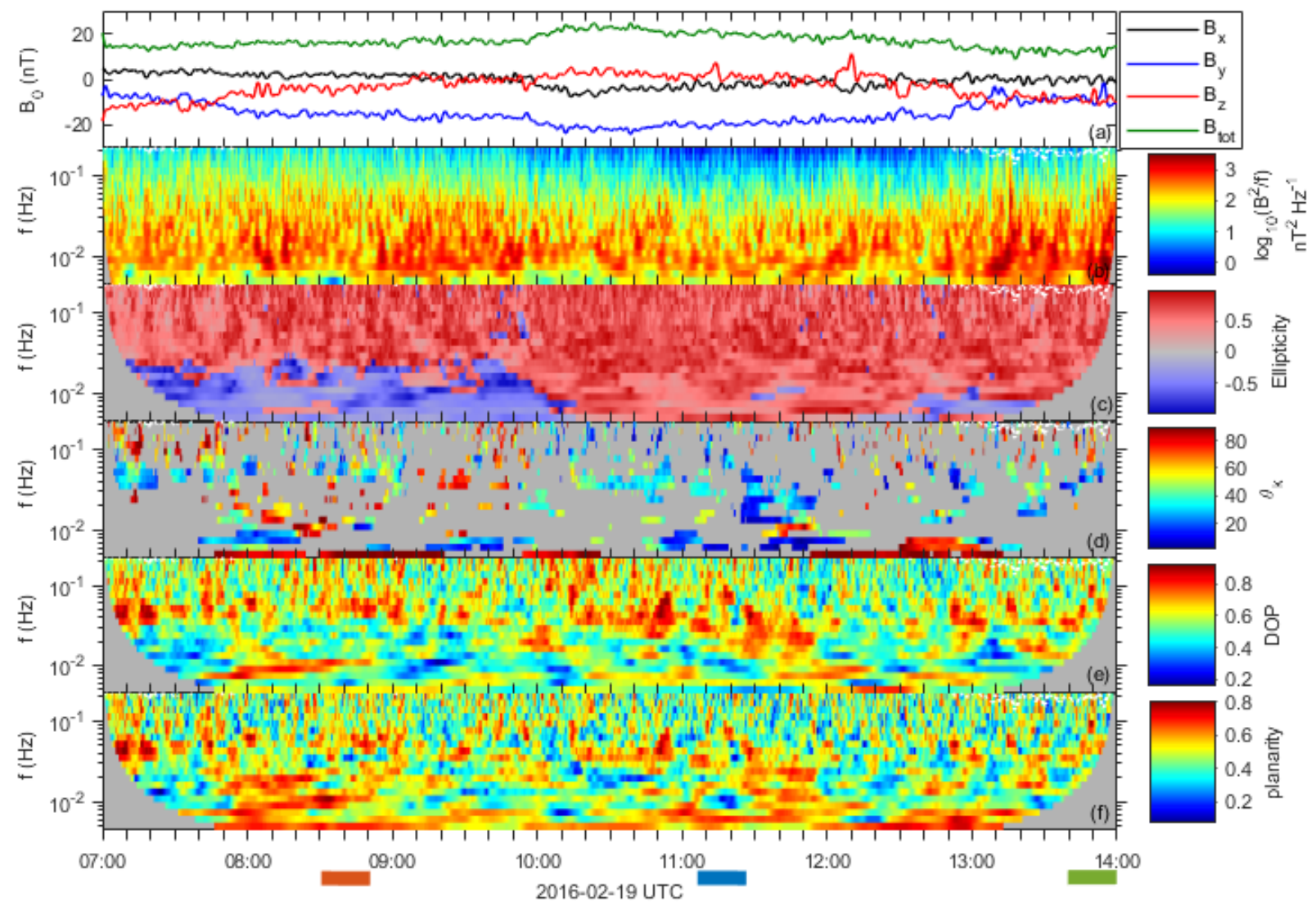

Fig. 4. Polarization analysis of the magnetic field for the time interval 07:00-14:00 UT on 2016 February 19. From top to bottom, panel a: 3D waveforms of the background magnetic field and its modulus, low-pass filtered at $2 \mathrm{mHz}$. Panel $b$ : spectrogram of the magnitude-squared total magnetic field. Panel $c$ : polarization ellipticity of the magnetic field vector. Panel $d$ : angle $\theta_{k}$ between the wave vector and the background magnetic field, in degrees. The wave vector is only drawn for a sufficient degree of polarization (DOP $>0.6)$ and planarity $(>0.6)$. Panel $e$ : degree of polarization of the magnetic field vector. Panel $f$ : planarity of the wave vector. The 20-min time intervals used to compute Fig. 3 are shown as horizontal solid lines below the time tags, using the same color code.

[10-30] $\mathrm{mHz}$ frequency range, it is clearly lower by a factor of 5-10 at higher frequencies. This is in good agreement with the weakening of the $\sim 10-100 \mathrm{mHz}$ magnetic field fluctuations previously noted in Hajra et al. (2017).

To study the magnetic field oscillations in more detail, we performed the polarization analysis of the 3D magnetic field waveform using the single-value decomposition (SVD) method (Santolik, 2003). This analysis is reported in Fig. 4 before and during the cometary outburst, that is, in the time interval 07:00-14:00 UT. The background magnetic field $B_{0}$, which is low-pass filtered at $0.002 \mathrm{~Hz}$ using a finite-impulse response (FIR) filter, and used to decompose the magnetic field fluctuations into parallel and perpendicular directions, is displayed in panel a. The rotation of the magnetic field observed during the outburst, described in Hajra et al. (2017), is also clearly seen here, whereas it is rather steady before the outburst. To take into account the possibility of a small residual offset and spacecraft residual field in the magnetic field RPC-MAG measurements, we applied an error propagation method (here $\pm 3 \mathrm{nT}$ on each of the three components) for every calculation in order to ensure the robustness of the analysis and of the results discussed below.

Using a Morlet wavelet transform algorithm (see, e.g., Grinsted et al. 2004), we computed the wave magnetic field ( $B_{\mathrm{w}}=B-B_{0}$ ) power spectrum, shown in Fig. 4b. This spectrogram shows steady magnetic oscillations in the frequency range [10-100 $\mathrm{mHz}]$ before the outburst occurring at 10:00 UT. The weakening of the wave magnetic oscillations during the outburst noted above is also observed close to $\sim 100 \mathrm{mHz}$.

The wave nature of this magnetic activity can be inferred from the degree of polarization (DOP) of the magnetic oscillations $B_{\mathrm{w}}$ displayed in Fig. 4e. DOP $>0.8$ in the frequency range $[\sim 10-100] \mathrm{mHz}$, that is, in the cometary ion mode frequency range, which means that these waves can be seen as highly polarized wave packets. These wave packets also have a planarity close to 1 , as can be seen from Fig. 4f, meaning that they can be considered as plane waves. Interestingly, these highly polarized wave packets are still observed during the outburst, but at lower frequencies $(\sim 20 \mathrm{mHz})$.

The $B_{\mathrm{w}}$ ellipticity is shown in Fig. 4c. It is defined as the ratio between the transverse components of $B_{\mathrm{w}}$, that is, when the ellipticity is equal to $1(-1)$, the wave is circularly right(left-) handed polarized, while when the ellipticity is equal to 0 , the wave is linearly polarized. From Fig. 4c, the cometary ion wave packets $(f \approx 50 \mathrm{mHz})$ are observed to be elliptically right-handed polarized, whereas a wave mode at lower frequency ( $f \approx 10 \mathrm{mHz}$ ) is predominantly linearly to left-handed polarized. This therefore suggests that two different wave modes coexist in the cometary ion wave frequency range. We note here that the frequency range of the two different wave modes decreases during the outburst, as noted above.

Finally, the direction of propagation of the wave packets is shown in Fig. $4 \mathrm{~d}$ through $\theta_{k}$, defined as the angle between the wave vector $\boldsymbol{k}$ and $\boldsymbol{B}_{\mathbf{0}}$. We observe that the propagation 
of cometary ion wave packets (in the frequency range $[\sim 40$ $60] \mathrm{mHz})$ is predominantly parallel $\left(\theta_{k} \approx 0^{\circ}\right)$ to oblique $\left(\theta_{k} \approx\right.$ $50^{\circ}$ ) before and during the outburst, but becomes mostly perpendicular to the magnetic field $\left(\theta_{k} \approx 90^{\circ}\right)$ just before the outburst at 09:10 UT (where $B_{z} \approx 0 \mathrm{nT}$ ). This change might result from the rotation of the magnetic field due to the local magnetic field draping that occurs during the outburst. However, this point is beyond the scope of this study and is left for future investigation.

\section{Correlation between magnetic field and plasma density oscillations}

Because of the high temperature ratio between cometary electrons and cometary ions $\left(T_{\mathrm{e}} / T_{\mathrm{i}}>100\right)$, cometary ions are too cold to significally contribute to the total plasma pressure. We therefore hereafter only consider the electron pressure contribution to the plasma pressure through the plasma density and the electron temperature. Considering the electrons isothermal, as discussed in Sect. 2, we make use of the new high timeresolution cross-calibrated density as an ersatz plasma kinetic pressure. In this section, we compute the correlation between magnetic and density fluctuations to study the balance between plasma thermal pressure and magnetic pressure of the cometary ion waves before and during the outburst.

This analysis is reported in Fig. 5, where the high timeresolution RPC-MAG and cross-calibrated plasma densities are investigated before (top) and during (bottom) the outburst on 2016 February 19. The waveforms of the relative variations of the magnetic field amplitude $(\delta|B| /|\langle B\rangle|)$ and cross-calibrated density $(\delta n /\langle n\rangle)$, with $\langle B\rangle$ and $\langle n\rangle$ the mean magnetic field and plasma density, respectively, over the considered time window, are presented in the top panel of each plot. In order to be able to compare them easily, the two signals were bandpass-filtered in their respective frequency range observed in Fig. 3, that is, [40-60] $\mathrm{mHz}$ and [20-30] $\mathrm{mHz}$.

For each time interval considered, we computed the magnitude-squared coherence of the magnetic field and density signals in the time-frequency plane, as shown in the bottom panel of each plot in Fig. 5. Using a Morlet wavelet, this method (Grinsted et al. 2004) measures the correlation (ranging from 0 to 1 ) between the two signals over the time interval at different frequencies. For areas where the coherence exceeds 0.5 , we added in this panel the phase difference (or lag) between these two signals computed from the wavelet cross-spectrum, shown by the black arrows. The direction of the arrows corresponds to the phase lag on the unit circle, that is, a horizontal arrow directed to the right (left) means a $0^{\circ}\left(180^{\circ}\right)$ phase lag.

Figure 5 shows clear coherence peaks (where coherence exceeds 0.5 ) of a few tens of seconds between the magnetic field and density signals in the [40-60] and [20-30] $\mathrm{mHz}$ frequency ranges, meaning that they are related. In these high-coherence areas, the black arrows are observed to be mostly directed to the right and almost horizontal, implying that magnetic field and density fluctuations are mostly in phase, which is confirmed by the waveforms in the top panels of Fig. 5. However, the two signals are also sometimes observed to be in antiphase (arrows directed to the left). Thus the phase lag between the plasma density and the magnetic field seems to oscillate between 0 and $180^{\circ}$ when the two signals are coherent.

In addition, our observations show that the magnitudesquared coherence between magnetic and density fluctuations usually coincides with a close-to-linear polarization of the wave. Thus, our understanding is that the wave quasi-linear polarization is associated with a compression of the plasma (i.e., the wave mode is compressional). We also note that when a quasi-linear polarization of the B-field is observed, its axis is usually oblique (with an angle of roughly $45^{\circ}$ ) to the background magnetic field (not shown here). This is true before and during the outburst. However, we have seen in Fig. 4 that the direction of propagation (wave vector) of the cometary ion mode is also oblique, with an average value of $50^{\circ}$. Thus, the polarization axis and the $\boldsymbol{k}$-vector may be aligned for the ion cometary mode. This might explain the plasma compression associated with the quasi-linear polarization observed above.

This result indicates that the cometary ion mode is compressional, which is consistent with the fact that a linear magnetic polarization is sometimes observed in Fig. 4. It also indicates that these waves do not disappear during the outburst and their properties remain unchanged, except that their carrier frequency decreases. The implication of these results is discussed in the following section.

\section{Discussion and conclusions}

In this study we used Rosetta in situ measurements to investigate the properties of the recently discovered low-frequency singing comet waves (Richter et al. 2015, 2016), also known as cometary ion mode (Meier et al. 2016). We took advantage of high time-resolution magnetic field measurements and a newly created high time-resolution density dataset obtained from the cross-calibration of RPC-MIP and RPC-LAP instruments, which allowed us to cover the frequency range of the cometary ion mode.

The main properties of the cometary ion waves in this study can be summarized as follows:

- they are observed in the typical frequency range $(\sim 50 \mathrm{mHz})$ before the cometary outburst;

- their carrier frequency decreases $(\sim 20 \mathrm{mHz})$ during the outburst and recovers its typical value after the outburst;

- the waves are observed to be highly polarized (DOP $>0.7$ ) and almost plane (planarity $>0.7$ );

- they are also observed to be elliptically right-hand polarized $(0<$ ellipticity $<1)$;

- they propagate mostly parallel to oblique $\left(\sim 0-50^{\circ}\right)$ to the background magnetic field;

- the wave mode is observed to be compressional before and during the outburst, and the phase lag between magnetic and density fluctuations oscillates between 0 and $180^{\circ}$;

- a lower frequency $(<20 \mathrm{mHz})$ wave mode is also observed, but its polarization is elliptically left-handed $(-1<$ ellipticity $<0$ ).

We here discuss the properties of the cometary ion mode in the light of these findings, and their implication on their generation mechanism.

First, we confirmed some of their properties shown in previous studies, such as their elliptical right-handed polarization (see, e.g., Koenders et al. 2016; Heinisch et al. 2017), and their propagation almost parallel $\left(\sim 0-50^{\circ}\right)$ to the background magnetic field, in agreement with the modified ion-Weibel instability (see Fig. 5 in Meier et al. 2016), but in contrast with observations by Richter et al. (2016). We also corroborated that their $\sim 50 \mathrm{mHz}$ frequency range is similar to what has previously been observed at different cometocentric distances (see, e.g., Richter et al. 2015) and derived from both the linear theory of the modified ion-Weibel instability (Meier et al. 2016) and solar wind-comet interaction simulations (Koenders et al. 2016). 

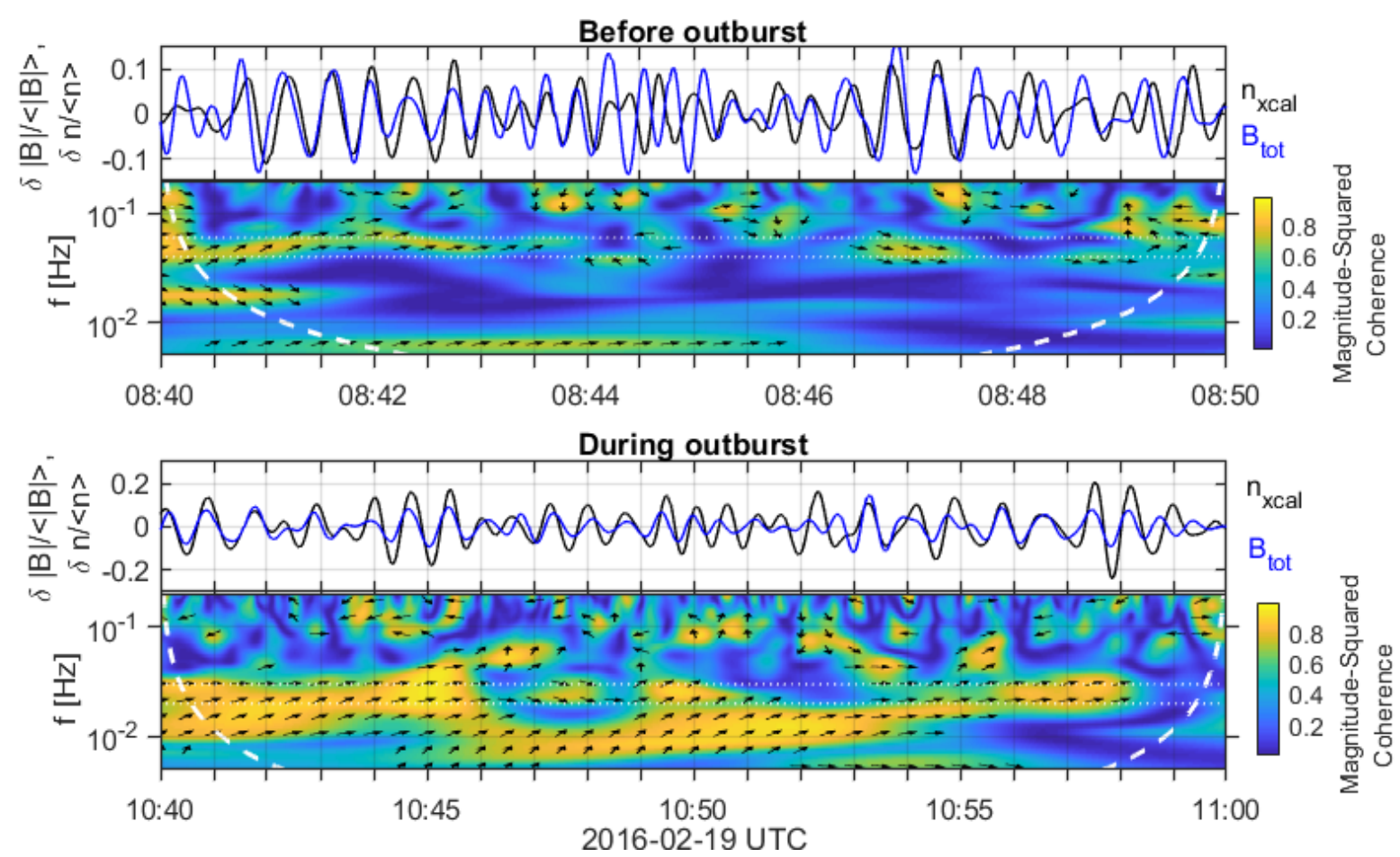

Fig. 5. Correlation of the relative magnetic field amplitude and plasma density oscillations observed before (top) and during (bottom) the cometary outburst on 2016 February 19. The top panels of each plot show the waveforms of the relative magnetic field amplitude $(\delta|B| /\langle|B|\rangle)$, blue line) and plasma density $(\delta n /\langle n\rangle$, black line) variations, bandpass-filtered in the [40-60] $\mathrm{mHz}$ (top) and [20-30] $\mathrm{mHz}$ (bottom) frequency range. The bottom panels show the magnitude-squared wavelet coherence of the magnetic field and cross-calibrated density signals in the time-frequency domain. The frequency range in which the signals, displayed in top panels, have been filtered is shown by the dotted white lines. The direction of the black arrows correspond to the instantaneous lag between the two signals on the unit circle.

Second, using a cometary outburst, we showed for the first time how their center frequency evolves as the plasma conditions changes, here with a decrease of about $20 \mathrm{mHz}$. However, their amplitude does not weaken and they do not disappear, as suggested in Hajra et al. (2017). We note that during the occurrence of this cometary outburst, the Rosetta orbiter spacecraft was located deep inside the inner coma, at about $35 \mathrm{~km}$ from the cometary nucleus, in a region where the cometary plasma is expected to experience a significant collisional coupling with the surrounding cometary neutral environment, with the ion exobase estimated to be located in the range $750-1500 \mathrm{~km}$ before and during the outburst, respectively, when computed from Eq. (2) in Mandt et al. (2016) using local parameters. We therefore suggest that the observed frequency decrease might be due to a deceleration of the water ions in the close plasma environment of the nucleus during the outburst. In order to validate this hypothesis, we evaluated the local change in the acceleration of the cometary water ions in this partially ionized medium, before and during the cometary outburst. To this purpose, we considered a toy model that mimics the competition between the (global) Lorentz force that accelerate cometary ions and a (local) ionneutral viscous drag that limits the cometary ion velocity, as in Eq. (9) in Simon Wedlund et al. (2017). We consider the magnetic field directed along the $y$-axis, the solar wind velocity $u_{\mathrm{SW}}$ is directed along the $x$-axis in the CSEQ frame and the electric field $E=0 \mathrm{mV} \mathrm{m}^{-1}$ (see Meier et al. 2016), so that the resulting acceleration takes place along the $z$-axis. Finally, considering the fact that ions will gyrate, but on a much larger scale, it is possible to simplify the latter expression as follows:

$m_{\mathrm{i}} \frac{\mathrm{d} u_{\mathrm{z}}}{\mathrm{d} t}=q_{\mathrm{i}} u_{\mathrm{SW}} B_{\mathrm{y}}-k_{\mathrm{i}, \mathrm{s}} m_{\mathrm{i}} n_{\mathrm{s}}\left(u_{\mathrm{z}}-v_{\mathrm{s}}\right)$, where $m_{\mathrm{i}}$ is the water-ion mass, $q_{\mathrm{i}}$ their electric charge, and $u_{\mathrm{z}}$ their radial velocity; $k_{\mathrm{i}, \mathrm{s}}$ is the $\mathrm{H}_{2} \mathrm{O}^{+}-\mathrm{H}_{2} \mathrm{O}$ ion-neutral drag rate (Gombosi 2015); $v_{\mathrm{s}}$ is the radial velocity of outgassing neutrals, and $n_{\mathrm{s}}$ is their density. We consider the following parameters: $k_{\mathrm{i}, \mathrm{s}}=1.5 \times 10^{-15} \mathrm{~m}^{3} \mathrm{~s}^{-1}$ and $v_{\mathrm{s}}=1000 \mathrm{~m} \mathrm{~s}^{-1}$ from Simon Wedlund et al. (2017), and $u_{\mathrm{SW}}=400 \mathrm{~km} \mathrm{~s}^{-1}$ and $B_{\mathrm{y}}=$ $2.5 \mathrm{nT}$ from Meier et al. (2016), where the waves are supposedly generated. Taking $u_{0}=v_{\mathrm{s}}$ as the initial condition, we then solved this equation for neutral densities observed before $\left(n_{\mathrm{s}}=0.75 \times\right.$ $\left.10^{14} \mathrm{~m}^{-3}\right)$ and during $\left(n_{\mathrm{s}}=1.5 \times 10^{14} \mathrm{~m}^{-3}\right)$ the considered outburst, as observed by the ROSINA/COPS instrument (Balsiger et al. 2007) on board Rosetta (see Fig. 1 in Hajra et al. 2017). The result of this integration is shown in Fig. 6a, where the velocity of water ions is drawn as a function of time. We show that when the plasma and neutral conditions at the location of Rosetta are considered, the water-ion velocity remains below an upper limit given by the asymptotic velocity $u_{\mathrm{z}} \simeq q_{\mathrm{i}} u_{\mathrm{SW}} B_{\mathrm{y}} /\left(k_{\mathrm{i}, \mathrm{s}} m_{\mathrm{i}} n_{\mathrm{s}}\right)$, which is estimated to be $\sim 47$ and $\sim 24 \mathrm{~km} \mathrm{~s}^{-1}$ before (blue line) and during (orange line) the outburst. Thus the water-ion velocity is assumed to be decreased by a factor 2 in this case.

We note here that the obtained asymptotic value of the waterion velocity is only valid at relatively short distances from the nucleus because our oversimplified model does not take into account plasma and neutral inhomogeneities around the comet and other acceleration processes associated with other contributions to the electric field (Madanian et al. 2016; Nilsson et al. 2018). However, it is assumed to be valid at the wave source because the waves are observed to grow relatively close $(\lesssim 500 \mathrm{~km})$ to the nucleus (Koenders et al. 2016; Meier et al. 2016). Moreover, this asymptotic value is close to an observational estimate from Nilsson et al. (2015). It is also in agreement 

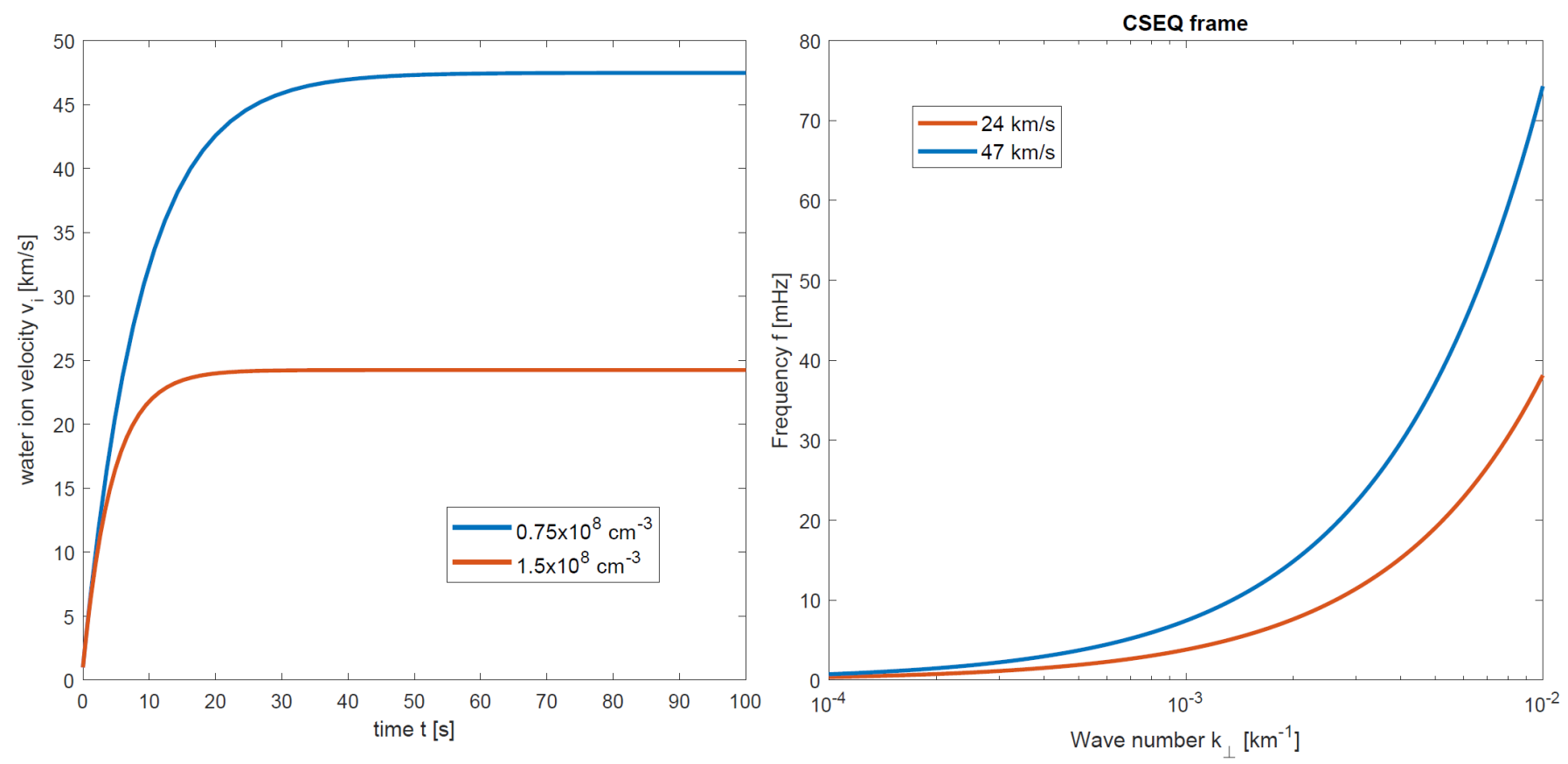

Fig. 6. Left panel: velocity of water ions as a function of time, obtained by solving Eq. (2), using the density of neutrals observed before (blue) and during (orange) the outburst. Right panel: dispersion relation of the cometary ion mode in the CSEQ frame following Meier et al. (2016) and using the bulk plasma velocity computed before (blue) and during (orange) the outburst (see left panel).

with hybrid simulations (see, e.g., Koenders et al. 2015, 2016). Therefore, our model illustrates how much the water-ion acceleration is expected to decrease in the vicinity of the nucleus during the outburst due to the friction with increasing outgassing neutrals, and provides a useful order of magnitude.

Following Richter et al. (2015) and Meier et al. (2016), we then estimated the real frequency of the unstable mode using the beam-mode dispersion relation (Chang et al. 1990), that is, the frequency resulting from the Doppler shift effect in the cometary rest frame (CSEQ) at the wave source, as follows:

$f=k_{\perp} u_{\mathrm{z}} \cos \left(\phi_{\mathrm{c}}\right) / 2 \pi$,

where $\phi_{\mathrm{c}}=\arctan \left(u_{\mathrm{z}} / u_{\mathrm{Sw}}\right)$.

The result is shown in Fig. $6 \mathrm{~b}$ for the two velocities before (in blue) and during (in orange) the outburst, as determined in the previous paragraph. Considering the wave number $k_{\perp, \max }=6.4 \times$ $10^{-3} \mathrm{~km}^{-1}$ for which the wave growth is maximum (see Meier et al. 2016), the frequency in the spacecraft frame in Fig. $6 \mathrm{~b}$ gives $\sim 47$ and $\sim 24 \mathrm{mHz}$ before and during the outburst, respectively. This is in good agreement with the magnetic field observations in Fig. 4. Similar results were also obtained by Meier et al. (2016, see Fig. 12 therein).

According to this figure, the frequency of the observed cometary ion waves does not scale as the local ion cyclotron frequency and therefore does not follow the variations of the local magnetic field magnitude, whereas it is expected from theory, see Meier et al. (2016). Instead, our study shows that the frequency of the observed cometary ion waves depends solely on the Doppler shift effect. Therefore, it is consistent with the idea that the waves are not generated locally but are convected by the bulk plasma.

These results are consistent with the modified ion-Weibel theory, in which these waves are zero-frequency wave modes that are generated farther away from the comet. Hence, although this is an oversimplified model that assumes cold, homogeneous three-component plasma, it enables us to show that the observed change in wave frequency is consistent with a Doppler shift change associated with the plasma flow slowing down because of additional friction with outgassing neutrals. Through this process, we argue that the change in frequency of the cometary ion waves could be a way to indirectly probe the water-ion velocity in its source region and thus the strength of neutral-plasma coupling in the environment of 67P. This result also demonstrates that the waves do not disappear during the cometary outburst, in contrast with the conclusions from Hajra et al. (2017), which ultimately implies that solar wind ions are still present during the outburst, at least in the wave source region, but are probably out of sight of the RPC-IES instrument at the location of Rosetta.

Then, we studied the correlation between plasma and magnetic oscillations before and during the outburst. Based on the newly created high time-resolution cross-calibrated plasma density dataset, we showed that in the cometary ion mode frequency range the magnetic field and density oscillations are mostly coherent or related, which is consistent with the observations of a partially linearly polarized magnetic field in this frequency band in Fig. 4. We also showed that the phase lag between magnetic and density oscillations varies from 0 to $180^{\circ}$, in agreement with theoretical calculations of dispersion relations for a two-ion plasma (Volwerk et al., priv. comm.).

To conclude, the measurements from multiple RPC instruments shown in this paper are consistent with the modified ionWeibel instability as the source of the cometary ion wave activity. We also argue that the observed frequency of the cometary ion mode could be a way to indirectly probe the strength of neutral-plasma coupling in the environment of 67P. Moreover, we showed that another intense, lower-frequency and left-handed polarized wave mode is observed at the same time. However, at such low frequencies, the magnetic field signal might also be related to or contaminated by spacecraft interference due to manoeuvres that occurred on that particular day. Therefore, this point is beyond the scope of the present study and is left for future investigation. 
Acknowledgements. Rosetta is an ESA mission with contributions from the member states and from NASA space agency. Work at LPC2E is supported by ESEP, CNES and by ANR under the financial agreement ANR-15-CE31-0009-01. The RPC-MIP/LAP cross-calibration work was funded by ESA through the RPC Enhanced Archive activity. We thank the ESA Planetary Science Archive (PSA) for archiving Rosetta-RPC instrument datasets. The work on RPC-MAG was financially supported by the German Ministerium für Wirtschaft und Energie and the Deutsches Zentrum für Luft und Raumfahrt under contract 50QP1401.

\section{References}

Acton, C. H. 1996, Planet. Space Sci., 44, 65

Balsiger, H., Altwegg, K., Bochsler, P., et al. 2007, Space Sci. Rev., 128, 745

Carr, C., Cupido, E., Lee, C. G. Y., et al. 2007, Space Sci. Rev., 128, 629

Chang, C. L., Wong, H. K., \& Wu, C. S. 1990, Phys. Rev. Lett., 65, 1104

Eriksson, A. I., Boström, R., Gill, R., et al. 2007, Space Sci. Rev., 128, 729

Glassmeier, K.-H. 2017, Phil. Trans. R. Soc. London, Ser. A, 375, 20160256

Glassmeier, K.-H., Boehnhardt, H., Koschny, D., Kührt, E., \& Richter, I. 2007a, Space Sci. Rev., 128, 1

Glassmeier, K.-H., Richter, I., Diedrich, A., et al. 2007b, Space Sci. Rev., 128, 649

Gombosi, T. I. 2015, Physics of Cometary Magnetospheres (Washington, D.C: American Geophysical Union), 169

Grinsted, A., Moore, J. C., \& Jevrejeva, S. 2004, Nonlinear Process. Geophys., 11,561
Grün, E., Agarwal, J., Altobelli, N., et al. 2016, MNRAS, 462, S220 Hajra, R., Henri, P., Vallières, X., et al. 2017, A\&A, 607, A34

Heinisch, P., Auster, H. U., Richter, I., et al. 2017, MNRAS, 469, S68 Heritier, K. L., Henri, P., Vallières, X., et al. 2017, MNRAS, 469, S118

Koenders, C., Glassmeier, K.-H., Richter, I., Ranocha, H., \& Motschmann, U. 2015, Planet. Space Sci., 105, 101

Koenders, C., Perschke, C., Goetz, C., et al. 2016, A\&A, 594, A66

Madanian, H., Cravens, T. E., Rahmati, A., et al. 2016, J. Geophys. Res. Space Phys., 121, 5815

Mandt, K. E., Eriksson, A., Edberg, N. J. T., et al. 2016, MNRAS, 462, S9

Meier, P., Glassmeier, K.-H., \& Motschmann, U. 2016, Ann. Geophys., 34, 691

Nilsson, H., Stenberg Wieser, G., Behar, E., et al. 2015, A\&A, 583, A20

Nilsson, H., Gunell, H., Karlsson, T., et al. 2018, A\&A, 616, A50

Odelstad, E., Eriksson, A. I., Edberg, N. J. T., et al. 2015, Geophys. Res. Lett., 42,10

Odelstad, E., Stenberg-Wieser, G., Wieser, M., et al. 2017, MNRAS, 469, S568

Odelstad, E., Eriksson, A. I., Johansson, F. L., et al. 2018, J. Geophys. Res. Space Phys., 123, 5870

Richter, I., Koenders, C., Auster, H.-U., et al. 2015, Ann. Geophys., 33, 1031

Richter, I., Auster, H.-U., Berghofer, G., et al. 2016, Ann. Geophys., 34, 609

Simon Wedlund, C., Alho, M., Gronoff, G., et al. 2017, A\&A, 604, A73

Taylor, M. G. G. T., Altobelli, N., Buratti, B. J., \& Choukroun, M. 2017, Phil Trans. R. Soc. A Math., Phys. Eng. Sci., 375, 20160262

Trotignon, J. G., Michau, J. L., Lagoutte, D., et al. 2007, Space Sci. Rev., 128, 713

Welch, P. 1967, IEEE Trans. Audio Electroacoust., 15, 70 